\title{
Amis et correspondants du CIBP
}

Constitution du bureau provisoire

Thérèse Goyet et Dominique Descotes

\section{OpenEdition}

Journals

Édition électronique

URL : http://journals.openedition.org/ccibp/395

DOI : $10.4000 /$ ccibp.395

ISSN : 2493-7460

Éditeur

Centre international Blaise Pascal

\section{Édition imprimée}

Date de publication : 4 janvier 1980

Pagination : 5-9

ISSN : 0249-6674

Référence électronique

Thérèse Goyet et Dominique Descotes, "Amis et correspondants du CIBP », Courrier du Centre international Blaise Pascal [En ligne], 2 | 1980, mis en ligne le 23 novembre 2015, consulté le 05 mai 2019. URL : http://journals.openedition.org/ccibp/395; DOI : 10.4000/ccibp.395

Ce document a été généré automatiquement le 5 mai 2019.

Centre international Blaise Pascal 


\title{
Amis et correspondants du CIBP
}

\author{
Constitution du bureau provisoire
}

Thérèse Goyet et Dominique Descotes

\section{Statuts déposés à la Préfecture du Puy-de-Dôme}

1 Il a été formé à la date du 13 décembre 1980 et sera soumis à la ratification de la première assemblée générale.

2 Présidente: $\mathrm{M}^{\mathrm{me}}$ Thérèse GOYET, Directeur Scientifique du CIBP et Professeur à l'Université Clermont II.

Vice-Président: M. Philippe SELLIER, Membre fondateur du CIBP et Professeur à l'Université Paris V.

Secrétaire : $\mathrm{M}^{\text {lle }}$ Marie-Thérèse SART, Conservateur en Chef de la BMIU.

Trésorière : $\mathrm{M}^{\text {lle }}$ Dominique SEGUIN, Bibliothécaire à la BMIU.

Trésorier-Adjoint: M. Dominique DESCOTES, Membre fondateur du CIBP et Assistant à l'Université Clermont II.

\section{Les résultats de l'enquête de fondation}

3 Nous parlons plus loin des fiches qui nous sont revenues, bibliographiques ou personnelles, et des ouvrages reçus en don. Ici nous présentons l'analyse des réponses au questionnaire en treize points qui avait été envoyé avec le Courrier nº 1, en 1979.

4 En cette première année d'existence le CIBP a reçu de vous quarante-cinq lettres de réponse. Nous présentons d'abord celles de leurs données qui sont quantifiables, question par question. Puis nous résumons et classons les principales suggestions, et alors les réponses peuvent interférer d'une question sur l'autre. Nous ajoutons, quand il y en a besoin, des indices distinctifs ( $a, b$, etc.) aux différents éléments des libellés. 


\section{Analyse quantitative}

QUESTION I. - Paraît-il utile?

- Un bulletin qui prolongerait par une information régulière la présente information vous.

oui 43 non 0 Abstentions 2

QUESTION II - Selon quelle périodicité ?

un an 16

six mois 13

trois mois 6

" pas trop souvent » 1

abstentions 9

QUESTION III - Quelle sorte de services en attendez-vous ?

- information bibliographique : oui : 44 ; non : 1 ; abst. : 0

- articles de fond : oui : 28 ; non : 12 ; abst. : 5

- revue de l'actualité pascalienne : oui : 36 ; non : 1 ; abst. : 7

- correspondance entre pascalisants : oui $: 28$; non :2 ; abst. : 13

QUESTION IV - Voir seconde partie.

QUESTION V - Avez-vous des articles prêts que vous souhaiteriez publier dans ce bulletin ? oui : 7 ; non 31 ; abst. : 6

QUESTION VI -

Sur le fonctionnement du CIBP. Doit-il devenir un fonds spécialisé permettant des recherches sur Pascal,

- recherches générales : oui : 37 ; non : 2 ; abst. 6

- spécialement orientées : oui : 25 ; non : 8 ; Abst. 12

Pour la $2^{\mathrm{e}}$ partie de la question, voir seconde partie de l'analyse.

Question VII - L'enrichissement de la Bibliothèque Municipale et Interuniversitaire, siège du CIBP, peut se faire en plusieurs domaines. Veuillez donner un numéro indiquant votre ordre de priorité aux rubriques suivantes :

a)- Premières éditions de Pascal.

b)- Études sur Pascal publiées jusqu’à maintenant.

c)- Collecte complète des éditions de Pascal.

d)- Livres connus de Pascal (originaux ou copies).

e)- Rassemblement des publications nouvelles en toutes langues.

f)- Collecte des traductions.

g)- Autres. 


\begin{tabular}{|l|l|l|l|l|l|l|}
\hline Nombre de voix & a & b & c & d & e & f \\
en $1^{\text {er }}$ position & 12 & 12 & 4 & 10 & 5 & 0 \\
en $2^{\text {e }}$ & 9 & 11 & 5 & 8 & 7 & 0 \\
en $3^{\text {e }}$ & 5 & 3 & 14 & 9 & 5 & 2 \\
en $4^{\text {e }}$ & 5 & 11 & 8 & 5 & 5 & 3 \\
en $5^{\text {e }}$ & 6 & 3 & 2 & 2 & 13 & 6 \\
en $6^{\mathrm{e}}$ & 1 & 1 & 3 & 2 & 1 & 23 \\
sans ordre indiqué & 0 & 1 & 1 & 1 & 0 & 0 \\
abstentions & 5 & 4 & 6 & 6 & 7 & 11 \\
\hline
\end{tabular}

La section g) est analysée dans la seconde partie.

QUESTION VIII - Voir seconde partie.

QUESTION IX - Souhaitez-vous que le CIBP parvienne, à un degré d'organisation tel qu'il puisse répondre par correspondance à vos questions concernant :

- sa propre documentation? oui : 43 ; non : 0 ; abst. 2

- la bibliographie pascalienne ? oui : 42 ; non : 0 ; abst. 3

- les activités d'autres chercheurs ? oui : 36 ; non : 2 ; abst. 7

Un non général : la réponse par le bulletin parait suffisante.

QUESTION X - Le CIBP pourrait promouvoir des rencontres d'études sur Pascal :

- sous forme de colloques pléniers, comme celui de 1976.

- sous forme de journées d'études à sujets restreints.

\begin{tabular}{|l|l|l|}
\hline Périodicité souhaitée & Colloques & \\
Un an & 2 & Journées \\
Deux ans & 4 & 10 \\
Trois ans & 3 & 6 \\
Quatre ans & 4 & 2 \\
Cinq ans & 10 & 2 \\
Six ans & 2 & 9 \\
Sept ou huit ans & 3 & \\
À ne pas préciser & 6 & \\
\hline
\end{tabular}

Sur les thèmes suggérés, voir seconde partie de l'analyse.

QUESTION XI - Le CIBP pourrait-il susciter des manifestations artistiques, qui accroitraient en quelque façon le rayonnement de Pascal?

oui : 26 ; non : 4 ; abst. 15

Pour les suggestions répondant à cette question, voir la seconde partie de l'analyse, de même que pour les questions XII et XIII.

II. - Analyse quantitative les suggestions dominantes. 
QUESTIONS VI - deuxième partie.

Quelles directions de recherches vous semblent-elles particulièrement indiquées?

QUESTION VII - Voir rubrique g question VI

5 On peut dire que les directions possibles ont toutes été rappelées, par un biais ou par un autre, ce qui prouve qu'aucun pascalisant ne pense que le programme des recherches pascaliennes soit achevé à l'heure où nous sommes, ou même puisse jamais être clos, la problématique se renouvelant par l'intégration des nouveaux acquis et par l'évolution des esprits. Entre toutes les virtualités constitutives de « la galaxie pascalienne », c'est donc l'engagement des personnes, ou des groupes de personnes, qui déterminera le choix des réalisations ; mais s'agissant du fonds matériel considéré comme instrument de travail propre au CIBP, deux lignes d'acquisition nous sont indiquées comme prioritaires par les réponses à la question VI et à la rubrique g) de la question VII. Ce sont d'une part la réunion des documents d'époque : sources supposées de Pascal, surtout dans le domaine scientifique, et œuvres des contemporains - que ce soit dans les originaux ou en reproduction - et d'autre part la documentation bibliographique. Comme moyen de tenir celle-ci à jour et en ouverture sur le travail qui se fait, on suggère de susciter pour chaque pays un correspondant qui fasse le dépouillement et l'adresse au Centre. Un soutien à ce recensement bibliographique est suggéré (en réponse à la question XIII) par l'établissement d'un répertoire des pascalisants, avec l'indication de leurs centres d'intérêt respectifs.

6 L'originalité du CIBP par rapport aux autres bibliothèques ou magasins de livres, devrait être son attention à collecter les informations les plus diverses relatives au milieu régional de Pascal. Une de ses utilités pourrait être de recueillir les travaux qui, n'étant pas imprimés, ne parviennent pas au dépôt légal, c'est-à-dire les thèses de troisième cycle et les mémoires, ou bien ceux qui périssent noyés dans la masse imprimée : coupures de journaux par exemple.

7 Citons pour résumer : « Le principe (du développement) devrait être de rendre aussi utile et attrayant que possible le séjour des chercheurs (français et étrangers) à Clermont ", ce qui postule « un véritable centre de documentation, à la limite, complet ».

QUESTION VIII - Dans le domaine audio-visuel, comment concevez-vous que le CIBP puisse utilement recueillir, conserver et faire connaitre les productions relatives à Pascal ?

Les réponses sont foisonnantes, comme le domaine en question! Mais les personnes qui seraient chargées de la conservation des documents et de leur mise à la disposition du public, font remarquer que ces opérations sont particulièrement difficiles. Ajoutons les achats particulièrement coûteux. Il faudrait donc souvent se contenter des renseignements imprimés (par ex. au moyen des revues spécialisées). Parmi les moyens les plus accessibles, la mini-cassette semble être celui qui recueille le plus d'intérêt. On pourrait aussi stocker des enregistrements très divers : manifestations en tout genre, séminaires, dictions exemplaires des textes de Pascal (ceci de grande utilité pédagogique). Certains de nos correspondants nous proposent ceux qu'ils possèdent.

Et bien sûr, on préconise le contact permanent avec les organismes de radio et de télévision et l'office du cinéma.

Pour les moyens seulement visuels, diapositives, photocopies, microfilms, ils ont été envisagés à la question VI. 
QUESTION X - Pour les colloques pléniers et les journées d'études, que souhaitez-vous comme thème?

- On souhaite une alternance entre ces deux types de rencontres.

- Il n'est pas nécessaire que, même organisés ou soutenus par le CIBP, les colloques se tiennent toujours à Clermont. L'essentiel est une idée organisatrice dont quelqu'un assume la responsabilité.

- Thèmes pour les colloques: selon les travaux en cours à présenter; selon les travaux publiés; réception et influence de Pascal ; Pascal savant; Pascal théologien; Pascal et la culture de son temps; son exégèse biblique.

- Pour les journées : "tables rondes" ou «séminaires» avec de véritables cours. Mêmes thèmes que pour les colloques; en plus limité ; les attaches avec la région d'Auvergne ; Pascal et Domat ; l'utilisation de tel ou tel prophète par Pascal ; étude de textes de Pascal délimités ; Pascal et le structuralisme ; Pascal et la psychanalyse ; alternativement - science et spiritualité ; la rhétorique de Pascal ; sa Vie par $\mathrm{M}^{\text {me }}$ Périer.

QUESTION XI - Les suggestions en matière de manifestations artistiques :

Le CIBP pourrait se borner à les accueillir ; ou à les susciter de loin par l'effet de son propre rayonnement. «Il semble (...) éphémère de vouloir aider au rayonnement de Pascal par un autre mode d'expression que celui dont Pascal même tire sa perdurance, à savoir l'écriture ». Expositions ; concerts ; ballets du XVII ${ }^{e}$ siècle ; spectacles théâtraux ; projections de films. Les expositions pourraient être itinérantes, et notamment, être prêtées aux ambassades. Excursions sur les pas de Pasca1. (Rejoint la question XIII).

QUESTION XII - Pour enraciner la connaissance de Pascal en sa région, quelles suggestions (méthodes de recherche, manifestations, etc.) proposez-vous?

- Certains craignent un rétrécissement d'horizon. D'autres préconisent des orientations multiples : « Il s'agit à la fois d'attirer dans la région les spécialistes de Pascal et d'intéresser le public de la région à Pascal. Pour les premiers, il faut des moyens de recherche; pour le second, des activités d'animation aussi attrayantes que possible ».

- Recherches sur les familles connues de Pascal.

- Édition de guides, cartes de la région au XVII ${ }^{\mathrm{e}}$ siècle ; un dépliant sur l'itinéraire pascalien en Auvergne, avec l'aide des syndicats d'initiative.

- Un fichier centralisant les nouvelles locales et les initiatives de recherche. Réunion de documents iconographiques.

- Excursions. Conférences.

- Interventions fréquentes dans la presse régionale.

QUESTION XIII - Autres suggestions d'activités.

Rejoignant la question XII, "l'inventaire et la conservation de tout ce qui, dans la région d'Auvergne, a, de près ou de loin, rapport avec Pascal ».

- Publication de nos travaux.

- Encourager des traductions dans des langues à diffusion restreinte.

- Bibliographie informatisée.

- Relation avec d'autres centres et avec les Universités.

- «La question unique est que Pascal soit lu». 
INDEX

Mots-clés : CIBP, statuts

AUTEURS

THÉRÈSE GOYET

CERHAC, Université Blaise Pascal

DOMINIQUE DESCOTES

CERHAC, Université Blaise Pascal 